\title{
Branching with Uncertain Semantics
}

Discussion Note on Saunders and Wallace, 'Branching and Uncertainty'

\section{Nuel Belnap and Thomas Müller}

\author{
1 Identifying the Problem \\ 2 Unraveling the Discussion \\ 3 Branching Structures \\ 4 Identifying the Grammar \\ 5 Identifying an Adequate Semantics of Branching Time \\ 6 Identifying the Saunders Wallace Proposed Semantics \\ 7 Conclusion
}

In their paper 'Branching and Uncertainty', (Saunders and Wallace [2008]) aim to solve the so-called 'incoherence problem' commonly attributed to the Everett interpretation of quantum mechanics (EQM). The Everett interpretation posits a multiplicity of branching universes as a realistic reading of the evolution of the quantum state function. The 'incoherence problem' is, roughly, that our common talk of uncertainty of outcomes of quantum-mechanical experiments seems to have no foothold in EQM: Since all the facts about the branching are fully acknowledged, there seems to be nothing, on that interpretation, to be uncertain about.

Saunders and Wallace point to non-epistemic approaches to the mentioned problem (e.g., Greaves [2004]; Greaves and Myrvold [2008]), but their own solution is instead to provide 'a ready set of semantic rules according to which our actual extant, ordinary talk of ignorance and uncertainty comes out as true' (pp. 293-4). Thus, there is no incoherence problem to begin with, just a question of getting the semantics right. This project, according to Saunders and Wallace, is not motivated by metaphysical considerations but by the hope of making sense of our 'best physical theory' along the lines of a naturalized metaphysics. 
We agree with much of what Saunders and Wallace are aiming at. Whether one's goal is to understand EQM or everyday indeterminism, branching is a basic concept that is needed to make sense of objective uncertainty about the future. $^{1}$

We agree and indeed insist that branching gives a perfectly coherent account of objective uncertainty. We take issue, however, with the way Saunders and Wallace carry out their task. The point of this discussion note is therefore partly critical and partly reconstructive. Our criticism is based on our sustained but unsuccessful attempt at identifying the 'ready set of semantic rules' promised in the paper's introduction. It isn't just that such rules are nowhere explicitly given in the paper: The scant exposition provided in the text glosses over a number of crucial choices that need to be made to identify such semantics, as is clear from extant discussion in readily available literature. Thus, even implicitly no rigorous semantic rules rules that at least in idealized cases measure up to the standard set by Tarski can be identified. Our reconstruction is founded on a mathematically exact semantic framework that has been proposed to explain objective uncertainty in terms of branching, namely, branching time. ${ }^{2}$ We hold that this framework, which is completely rigorous, preserves enough of the authors' central ideas to count as a friendly reconstruction serving their purpose of countering the incoherence problem of EQM.

\section{Identifying the Problem}

The problem of making sense of objective uncertainty has been discussed in at least two different areas of philosophy, both of them rather technical: the philosophy of physics and philosophical logic. There has not been much interaction between these two strands of discussion.

1 When we speak of 'objective uncertainty', we mean to characterize a mind-independent situation or event, as in the phrase, 'the situation is uncertain' or 'the outcome is uncertain'. As variants, we speak sometimes of 'indeterminism' and sometimes of an 'open future'. Using 'possible' in its mind-independent sense, by 'objective uncertainty' we intend to describe a situation or event that has multiple individually possible but pairwise impossible outcomes. The contrast between objective and subjective uncertainty perfectly agrees with the more familiar contrast between objective and subjective probability.

2 We have in mind 'branching time' as presented in (Belnap et al. [2001]) (henceforth FF), (Belnap [2002]) (henceforth DTR), and (MacFarlane [2003], [2005]). Its presentations elsewhere as mere mathematics are of no help, and others, such as that of (Lewis [1986]), are conceptually flawed. The mentioned publications should cause the authors to retract their claim that nobody apart from physicists has ever looked at branching without suspicion. We also wish to point out that the authors' claim that weighted branching is unheard of in the philosophical literature will have to be dropped when confronted with, e.g., (Belnap [1992]; Weiner and Belnap [2006]; and Müller [2005]). (The label 'branching time' is unfortunate. For reasons that will become clear later, 'branching histories' would be more appropriate. The label is, however, so deeply entrenched in the literature that we will continue to use it as well.) 
The problem that Saunders and Wallace are facing arises in the context of the interpretation of quantum mechanics: Given EQM, we seem to be in a situation where everything there is to be known is known (because the evolution of the quantum state describes all the facts about future branchings exactly), and still there is something we don't know (because intuitively, we are facing an uncertain future; cf. p. 301). This tension has suggested to many that either EQM must be dropped or our attitude toward the future will need to be redescribed in non-epistemic terms, accounting for what we do while denouncing our intuitive explanation of why we do what we do, which points to an objective uncertainty that EQM apparently disallows. Saunders and Wallace reject both these options: EQM is taken to be 'our best physical theory' (p. 294), ${ }^{3}$ and our stance toward the future is viewed as robustly grounded in objective uncertainty, ruling out a non-epistemic reinterpretation. Thus, the challenge is to find a place for objective uncertainty in a branching framework. In other words, the incoherence problem discussed by Saunders and Wallace has the following form.

\begin{abstract}
How can we give a coherent view of what the world is like that incorpo rates two facts forced upon us by our best physical theory: (a) a realistic interpretation of QM via EQM shows that we need to give a branching account of the evolution of the universe, (b) as practicing physicists we face objective uncertainty, e.g., in connection with the outcomes of quan tum mechanical experiments.
\end{abstract}

Worries about EQM were certainly not what motivated the logicians' tenselogical theories of branching time. Instead, from Aristotle's famous discussion of 'the sea-battle tomorrow' onward, the worry concerned the following.

Given our everyday intuitions about objective uncertainty, how can we find an appropriate semantic framework for the future tense of natural language?

Branching time is precisely such a framework. Viewed from this perspective, the challenge posed by EQM may sound somewhat surprising. After all, the incoherence problem would seem to vanish once one adopts the branching-time semantic framework. In fact, although they do not mention 'branching time', this is more or less what is said in Saunders and Wallace's paper. It could, however, be said more clearly, rigorously, and with ready references to existing literature, thus enforcing rather than weakening the connection between philosophy of physics and formal logic. This is what we aim to do in the rest of this discussion note.

3 It might be more congenial to call EQM an interpretation of QM, which is indeed one of our best physical theories. We won't take issue with this point here. 


\section{Unraveling the Discussion}

Saunders and Wallace start to discuss branching in the context of personal fission, taking up an argument between (Parfit [1976] and Lewis [1976]).

Whatever the merits of such a discussion, it is clearly not pertinent to the matter at issue, which is the objective uncertainty of the outcome of, e.g., a physical experiment. The authors themselves come to this conclusion when they explicitly suggest shifting the discussion of branching from intra-world branching of persons to branching of 'worlds as wholes' (p. 297), where by 'worlds' they presumably mean possible courses of events, i.e., what we call 'histories'. ${ }^{4}$ The move from persons branching to histories branching is good: Many examples of objective uncertainty discussed in the literature do not involve persons at all, and even though many do so, they are not needed for illustrating either objective uncertainty or EQM. Discussion of the mind-dependent features of persons facing an 'open future' brings in many difficult issues that are best dealt with after the notion of objective uncertainty has been clarified. ${ }^{5}$

Saunders and Wallace's next step is to address and dismiss Lewis's arguments against branching. While we refrain from endorsing a number of details in their argumentation, their conclusion, which is that Lewis gives only faulty arguments against branching, agrees with ours. ${ }^{6}$ Before going on to discuss the merits of their proposal for the understanding and defense of EQM, Saunders and Wallace wrap up their analysis by discussing the example sentence, 'Al Gore might have won the 2000 US presidential elections' (p. 298). On their view, that sentence is true if Al Gore 'has a temporal part which is part of a person who won' (p. 298). They later stress that a temporal part is a part of many histories, each a single course of possible events, while a personal continuant occurs in only one history.

On our view, it is good that Saunders and Wallace stress the importance of small entities for clarifying the semantics of such modal talk, and it is also

4 For the formal definition of 'history,' see Section 3 below. Lewis uses the term 'world' to denote a totality closed under 'suitable external relations' (Lewis [1986], p. 208), paradigmatically spatiotemporal and causal relations. In this sense of the term, in the use of which we follow Lewis, a totality of many branching histories (each a maximally consistent course of events) is a single world. Worlds cannot branch from each other since branching is evidently a 'suitable external relation.' When they use the phrase 'branching worlds', Saunders and Wallace, following Lewis, obliterate the crucial distinction between histories and worlds. In interpreting Saunders and Wallace, we will speak about 'branching histories' instead.

5 Such discussions tend to become entangled in difficult issues of, e.g., first-person thoughts and the nature of self-locating beliefs. We emphatically agree that in this area, there are challenging philosophical problems that need to be addressed, but in the interest of clarity, it seems wise to try and solve the base case first.

6 We submit that FF, pp. 205 9, clearly and conclusively refutes Lewis on this issue.

7 Cf. also p. 301, where they illustrate what they are after by the phrase 'big bang to end-of-time'. See note 4 above. 
good to allow these entities to occur in many histories. ${ }^{8}$ Where we disagree is with respect to the analysis of continuants. Saunders and Wallace do not make it easy to distinguish a possible 'life history', which by definition does not contain incompatible events, from a person (who may on occasion face future possibilities that are incompatible). With respect to the example, we would like to say the following: Al Gore might have won the 2000 US presidential elections if and only if in his past life, there was an event such that at that event, it would have been true to say 'It is possible that he will win', i.e., if there is at least one history passing through that event on which he later wins the election. ${ }^{9}$ All the time, we are talking about the man Al Gore, whose possible life histories branch, even though it makes no sense to say of the person, Al Gore, that he branches.

At any rate, for the basic point at issue, i.e., how to make sense of objective uncertainty against a branching background, an analysis of continuants would require an additional exploration, whereas examples employing just impersonal events appear to suffice. The first step in giving a semantic account suitable for EQM, which is the express purpose of the Saunders and Wallace paper, should therefore avoid requiring consideration of persons and life histories. The point is to adhere to Tarskian standards of rigor, being explicit and mathematically precise about the semantic rules. That this is possible for an assemblage of branching histories, each taken as a maximally consistent course of events, is witnessed by a number of publications. ${ }^{10}$ Saunders and Wallace, however, while referring to 'their semantics' many times, are never explicit. This would be sufficiently unhappy if their purpose were to clarify natural language, but the lack of rigor stands out especially in the present case, in which the concern is to illuminate a physical theory that from the beginning is phrased in mathematical language.

\section{Branching Structures}

There is a variety of rigorous semantics for a language in which one can express objective uncertainty. Common to many of them is the reliance on branching structures. Formally, a branching structure is minimally a strict partial ordering $\langle W,<\rangle$ (i.e., $W$ is a non-empty set and $<$ is a transitive and irreflexive and therefore asymmetric ${ }^{11}$ binary relation on $W$ ) that also satisfies the principle of no backward branching (i.e., for any $m_{1}, m_{2}$, and $m_{3} \in W$, if $m_{1}<m_{3}$ and $m_{2}<$ $m_{3}$, then either $m_{1}=m_{2}$ or $m_{1}<m_{2}$ or $\left.m_{2}<m_{1}\right)$. A history, $h$, is defined as a

8 For 'serviceability' rather than 'metaphysical principle' (p. 294), we even countenance point-like event-type entities.

9 In the tense-modal notation of FF, Chapter 8, explained in Section 5 below, we would say ' Was: Poss: Will:(Gore wins)'.

10 See, e.g., the references given in note 2 above.

11 That is, for no $m$ and $m^{\prime}$ do we have both $m<m^{\prime}$ and $m^{\prime}<m$. 
subset of $W$ that is linearly ordered by < (so that any two elements of $h$ are comparable) and that is maximal with respect to that property.

The elements of $W$, which we call moments, can be taken to carry various sorts of event-like information. In line with Prior's original idea of a branchingtime framework for discussing indeterminism (Prior [1967], pp. 126 7), moments could carry information about the facts everywhere across a universewide possible course of events at a single point in time what (Thomson [1977]) calls a (momentary) 'super-event'. We use $m$ to range over moments. For the ordering relation $m_{1}<m_{2}$, a temporal modal causal interpretation is natural: $m_{1}<m_{2}$ may faithfully be read as either ' $m_{1}$ is in the (settled) causal past of $m_{2}$ ' or ' $m_{2}$ is in the future of possibilities of $m_{1}$.' A history, $h$, is a concrete course of events, explicating the modal structure: Two moments are consistent if and only if there is a history to which they both belong.

Example 1. Let there be four moments in all: $W=\left\{m_{0}, m_{1}, m_{1}^{\prime}, m_{2}\right\}$, with $m_{0}<m_{1}<m_{2}, m_{0}<m_{1}^{\prime}$, and two histories, $h_{1}=\left\{m_{0}, m_{1}, m_{2}\right\}$ and $h_{1}^{\prime}=\left\{m_{0}, m_{1}^{\prime}\right\}$.

So $m_{1}$ and $m_{1}^{\prime}$ are inconsistent, as are $m_{2}$ and $m_{1}^{\prime}$.

In short, the branching of histories gives a formal representation of the alternative possibilities that form the basis for objective uncertainty.

It is consistent with the spirit of branching to suppose that each possible moment, $m$, can be assigned a unique positive real number as its date time, say date time $(m)=\rho$, in a partly conventional but also partly natural way. Using $<_{R}$ for the standard linear ordering of the reals, it is, e.g., a necessary (but not yet sufficient) condition on the function date time is that if $m_{1}<m_{2}$, then date time $\left(m_{1}\right)<_{R}$ date time $\left(m_{2}\right) .{ }^{12}$ Let $T$ be the range of the date time function, and let $<_{T}$ be $<_{R}$ restricted to $T$. Call $\left\langle W,<, T,<_{T}\right.$, date time $\rangle$ a 'BTDT' structure (branching times with date times). If one takes this route, one must be careful to distinguish date times in $T$ from moments in $W$. We may enrich Example 1 by adding some date time information.

Example 2. Add to Example 1 the following: $T=\left\{t_{0}, t_{1}, t_{2}\right\}$ with $t_{0}<_{R}$ $<t_{1}<_{R} t_{2}$ and date time $\left(m_{0}\right)=t_{0}$, date time $\left(m_{1}\right)=$ date time $\left(m_{1}^{\prime}\right)=t_{1}$ and $\operatorname{date} \operatorname{time}\left(m_{2}\right)=t_{2}$.

12 Our principal purpose here is merely to draw attention to the sharp distinction between moments and date-times; a second purpose is to counter the impression that use of date-times somehow presupposes determinism. These purposes fortunately do not require us to discuss the question of how date-times might be assigned in a useful way. See FF, Section 7A.5, for a closely related and more detailed treatment. 
Two easy and relevant remarks that are, however, also easy to forget: (i) Although the ordering of $W$ is tree-like, it is natural that the set, $T$, of date times admits a linear order such as $<_{R}$. (ii) That date time $\left(m_{1}\right)=$ date time $\left(m_{1}^{\prime}\right)$, even though $m_{1}$ is inconsistent with $m_{1}^{\prime}$, is nothing special. It is, however, misleading to report this situation by saying that 'even though they are inconsistent, $m_{1}$ and $m_{1}^{\prime}$ occur at the same time.' That recipe neglects that we are speaking of individually possible but jointly inconsistent moments, whereas 'occurs at the same time' wrongly suggests joint consistency. Better instead to endure the cumbersome ' $m_{1}$ and $m_{1}^{\prime}$ are alternative possibilities for the same time.'

With respect to EQM, a BTDT structure, $\left\langle W,<, T,<_{T}\right.$, date time $\rangle$ would most probably correspond to a branching tree of quantum states of the universe, even though the idea of continuous branching that is sometimes evoked in connection with EQM (e.g., Wallace [2008], p. 26) might pose formal challenges (we'll leave these considerations by the wayside). ${ }^{13}$

Based on a branching structure, semantical rules for interpreting sentences of a given language may be formulated. In what follows, we will only consider formal languages. ${ }^{14}$

\section{Identifying the Grammar}

Which (formal) language is appropriate for discussing and possibly resolving the incoherence problem for EQM?

Given the basic branching-time structure $\langle W,<\rangle$, it is possible to specify a number of different semantics for a basic propositional temporal modal language, i.e., a language containing propositional constants $(p, q, r, \ldots)$, propositional connectives (not, and, or, if ... then, often symbolized as $\neg, \wedge$, $\vee, \rightarrow$ ), temporal operators it was the case that and it will be the case that (symbolized by Prior as $P$ and $F$, and by FF as Was: and Will:), and the modalities of settledness ('historical necessity') and historical possibility (symbolized by FF as Sett: (short for 'settled true') and Poss:). It is this simple grammar that we

13 Evidently, BTDT is non-relativistic; it even lacks a concept of spatial or space-like separation. For an application of branching structures to physical theories, however, it seems that an explicit representation of space will be definitely beneficial and most probably necessary. We leave out discussion of the one extant formally rigorous framework that integrates branching and space in a relativistic way, namely, branching space times, in order not to complicate matters, and because Saunders and Wallace obviously do not consider that framework. See (Belnap [1992]) for the details. In such a relativistic framework, the notion of a date-time is replaced by the notion of spatiotemporal location; see (Müller [2005]) for details.

14 Saunders and Wallace might ultimately be interested in giving a semantics for natural language rather than for some formal language. It is clear, however, that there is no hope of providing semantics for any decent portion of natural language based on anything as frugal as a branching structure (consider propositional attitudes, mass terms, verbal aspect, adverbials, or your other favorite formal semanticists' nightmares). It seems therefore best to stick with formal languages, which may be viewed as subsets of, or models or idealizations of, a natural language. 
will take as our principal target in making a case that branching time, when properly understood, supports the coherence of EQM. Given a full BTDT structure, $\left\langle W,<, T,<_{T}\right.$, date time $\rangle$, it is also enlightening to include forms $A t_{t}: A$, where $A$ is a sentence and $t$ is an individual constant that 'rigidly' denotes a member of $T$, i.e., a date time. $A t_{t}: A$ is given the informal reading, 'that $A$ is true at date time $t$ ' ${ }^{15}$ For a richer language, typically only a subset of its expressions will admit of semantic interpretation given just a branching structure with date times.

Saunders and Wallace evidently have in mind a language whose grammar allows one to talk about individuals (e.g., events, persons, and so on) such as the language of predicate logic; such a language obviously needs additional information to allow for semantic interpretation. Formally, this points to the many issues of quantified modal logic, for the illumination of which a number of widely different semantic approaches have been proposed. A useful summary of such options is given by (Garson [2006]). An especially delicate issue in this area is the formal representation of individuals. Even deciding on an underlying branching structure still allows for many options in this respect. ${ }^{16}$ We will not consider individuals in what follows, for the reasons given at the end of Section 2.

\section{Identifying an Adequate Semantics of Branching-Time}

We think that the semantics of FF (Chapter 8) and DTR is of major help in understanding EQM. Here, we give a relaxed account of the semantics, trusting the reader to consult the mentioned sources for mathematically exact formulations. A critical requirement in giving a semantics based on a branching structure is to identify the index of evaluation, i.e., the suite of parameters with respect to which truth values of utterances are to be assessed. Following the ideas of (Kaplan [1989], pp. 591 3), such an index usually comprises both context parameters, such as moment, place, and speaker of the context of utterance, and auxiliary parameters that may be shifted by, for example, the modal and tense operators. ${ }^{17}$ For simplicity, we omit every element of context, except the moment of utterance. ${ }^{18}$ Even in non-branching tense logic,

15 There is an example involving $A t_{t}$ : in Section 5. Both here and there, we abuse notation by using ' $t$ ' sometimes as a date-time and sometimes as the name of a date-time.

16 We tend to favor an account of the semantics of singular terms in the manner of (Bressan [1972]) or (Gupta [1980]). See (Belnap [2006]).

17 For an overview of parameters of truth, cf., e.g., (MacFarlane [2005]; and Belnap [2007]). We avoid consideration of 'structure' and 'interpretation' parameters by supposing that we are dealing with a 'standard interpretation'.

18 If we were to consider sentences with 'I', we would need to invoke 'the speaker of the context of utterance' and thus to have to consider the various treatments of persons in branching time. Analogously, if we considered sentences with 'here', we should have to worry about places. These complications, which we take to be not directly relevant to the incoherence problem, may be postponed. 
the index of evaluation needs at least two parameters: one context parameter, 'the moment of the context of utterance', $m_{\mathrm{C}}$, and one auxiliary parameter, 'the moment of evaluation', $m .{ }^{19}$ Each must be an element of $W$. The former pertains to the moment at which a stand-alone sentence is uttered, presumably as the vehicle of some speech act. The auxiliary parameter, $m$, identifies a 'moment of evaluation', that is, an element of $W$ with respect to which embedded parts of uttered sentences are evaluated and which is shifted up and down a single history by tenses or by an $A t_{t}$ : operator. For example, the Was: operator shifts that parameter backwards in time so that for the sentence it was the case that $p$, the embedded $p$ is evaluated, as it should be, not at the moment of evaluation of the whole sentence but at some moment before that. The truth value of a stand-alone sentence must not depend on the value of auxiliary parameters: Such parameters must in effect eventually be 'canceled' either by quantifying over them or with the help of some element of the context. (This is nothing specific to modal languages: Standard first-order logic treats the quantificational auxiliary, 'assignment of values to the variables', $\alpha$, as something that needs to be canceled by quantification.) Given a standalone sentence of our language, the auxiliary moment of evaluation, $m$, is canceled in the second way, by identification with the moment of utterance, $m_{\mathrm{C}}$. That is to say, a stand-alone sentence is to be evaluated at a suite of parameters such that the moment of evaluation is identical to the moment of utterance: $m=m_{\mathrm{C}}$. We may say that the auxiliary moment parameter is 'canceled by initialization by context'. When there is no branching, there is no point in distinguishing moments and times. So much for non-branching: The required suite of parameters must be $\left\langle m_{\mathrm{C}}, m\right\rangle$.

In giving semantics for the future tense based on branching structures, there is no getting around the idea of a history, $h$, namely, a maximal linear chain within $W$ representing one complete possible course of events. The literature reveals several ways in which histories can play a role in the semantics.

1. (Prior [1967], pp. 128 36) describes the so-called 'Peircean' semantics, in which the future operator is taken to quantify over all histories, $h$, passing through the moment of evaluation, $m$, thus reading 'it will be the case that' as 'it is unpreventable that it will be the case that', or, to use the Thomason ([1970]) terminology, 'it is settled that it will be the case that'. On the Peircean semantics, there is quantification over histories, but there is no history parameter: Truth is relative simply to the pair, $\left\langle m_{\mathrm{C}}, m\right\rangle$. Although simple, Peircean semantics will do neither for EQM nor for our ordinary employment of the future tense in the context of indeterminism. With regard to the latter, 'I now

19 'The moment of the context of utterance' is such a mouthful that we shall say 'the moment of utterance' or 'the context moment'. 
bet that the outcome of the measurement will be spin-up' is not plausibly construed as a bet that it is now a settled matter that the outcome will be spin-up.

2. 'Occamist' semantics, also mentioned by (Prior [1967], pp. 121 7), employs an auxiliary history parameter, the 'history of evaluation', and takes truth to be relative to a triple $\left\langle m_{\mathrm{C}}, m, h\right\rangle$ (moment of utterance, moment of evaluation, and history of evaluation), where $h$ must contain $m$; Was: and Will: shoot existentially, respectively, backward and forward along $h$. This turns out to allow for a more natural reading of the future tense in both ordinary and EQM employment. Being auxiliary, the history of evaluation parameter must eventually be canceled. ${ }^{20}$ The literature has considered various possibilities.

2a. In the triple $\left\langle m_{\mathrm{C}}, m, h\right\rangle$, one might be tempted to take the auxiliary, $h$, to be canceled through unique determination by the moment of utterance, $m_{\mathrm{C}}$, or (which comes to the same thing) one might simply take $h$ itself to be a contextof-utterance parameter, or one may take the history, $h$, to be uniquely determined by the moment of evaluation, $m$. Each of these alternatives amounts to postulating that there is the actual future which is uniquely determined by a single momentary event. Each of these alternatives must be dropped because each is really just determinism in sheep's clothing. These views are discussed and criticized in Chapter 6 of FF under the heading, 'the thin red line'. ${ }^{21}$

2b. If the history of evaluation parameter, $h$, is not determined by context, then it is an auxiliary parameter and as such must eventually be canceled. There are many possibilities (see DTR); we mention just two.

2b1. (Thomason [1970]) cancels the history parameter in the service of a supervaluational theory of truth. Thus, leaving out $m_{\mathrm{C}}$ for the nonce, a sentence is said to be true [false] at a moment, $m$, just in case it is true [false] at every moment history pair $\langle m, h\rangle$; with $m \in h$, that is, just in case it is settled true [false] at $m$. This supervaluational lapse from bivalence answers to the idea that a sentence in the future tense may be neither settled true nor settled false when uttered. We recommend keeping the idea, which goes back to Aristotle, but not keeping the shortening of 'settled true' to plain 'true,' a

20 Apart from FF, it is difficult to find an Occamist account of branching time that explicitly recognizes this requirement. In its absence, writers are driven to characterizing $h$ as something like the history 'one has in mind', or 'the actual history' (e.g., Venema [2001]), neither of which makes more sense than characterizing an assignment, $\alpha$, of values to the variables as 'the assignment one has in mind' or 'the actual assignment'. The parallel, emphasized in FF, Chapter 6 , is perfect.

21 We think that regardless of one's views on 'content', whether externalist, functionalist, wide, narrow, or whatever, an uttering (or a thinking) is a concrete event which cannot uniquely determine a unique course of events up to and beyond the death of the sun. The discussion of Saunders and Wallace on p. 295f. misses the mark. An alternative interpretation of their proposal, which would, however, run counter to the explicitly stated aim of their paper, would be to say that branching doesn't play a role for their semantics after all. Such a reading would seem to us to be uncongenial. 
shortening that makes it almost impossible to avoid confusion. Think of the confusion in modal logic were one to uniformly shorten 'necessarily true' to plain 'true'. To fail to make the modality explicit in either case invites wholly unnecessary confusion. ${ }^{22}$

2b2. A more sophisticated use of the supervaluational idea is to distinguish the moment of evaluation of a future tense sentence from the moment defining the set of histories required for settledness. The intuition is that an utterance of a sentence at $m_{\mathrm{C}}$ (paradigmatically in the future tense) may well not have a settled truth value at $m_{\mathrm{C}}$, when it is uttered, even though it will be settled one way or the other later, a phenomenon labeled a 'double time reference' in DTR. This application of the semantics for branching time can make sense of our ordinary employment of predictions, as well as of promises, bets, and so forth, as subject to normative assessment. The key idea is that the auxiliary moment of evaluation, $m$, is canceled by identification with the moment of utterance, $m_{\mathrm{C}}$, whereas the auxiliary history of evaluation, $h$, is canceled by quantification with respect to all histories passing through a later 'moment of assessment' (the phrase is due to MacFarlane, ibid.). This makes it easy to see a rigorous and coherent semantic underpinning for sentences such as the following, which in English are almost hopelessly confusing to those lacking a firm grasp of the semantics required by indeterminism:

Example 3. ${ }^{23}$ As a matter of settled fact, it is (at $m_{2}$ ) false that 'The electron will be measured spin up' was (at $m_{0}$ ) settled (i.e., on both $h_{1}$ and $h_{1}^{\prime}$ ) true at any moment (i.e., $\left.m_{0}\right)$ prior to the moment of measurement $\left(m_{1}\right)$. But now (at $m_{2}$ ), it is a settled fact (i.e., on $h_{1}$, which is the only history through $m_{2}$ ) that 'The electron will be measured spin up' was true at each moment (i.e., $\left.m_{0}\right)$ prior to the moment of measurement $\left(m_{1}\right)$.

In accord with the treatment of 'double time references' in BTDT semantics (we'll call the whole package 'BTDT DTR semantics'), what must be done is to distinguish the following two, when asserted at some moment of assessment $\left(m_{2}\right)$ after the measurement came out 'spin up' (at $\left.m_{1}\right)$, with reference to a past moment of utterance $\left(m_{0}\right)$ of ' Will: $U p$ ' that is prior to the measurement:

Was:Sett:Will:Up (false) from Sett:Was:Will:Up (true). ${ }^{24}$ We could describe the situation as follows as a repetition and enrichment of previously displayed examples.

\footnotetext{
22 The sin is all too common; see, for example, the otherwise helpful (MacFarlane [2003]).

Parenthetical references are to Example 1.

24 It is essential to appreciate that although we have omitted nearly all hen-scratches, our words have exact meanings, as given in FF and DTR.
} 
Example 4. Assume, as in Examples 1 and 2, that there are just four moments, two histories, and three date times. The 'spin' facts are that the electron is measured spin-up at the possible moment $m_{1}$, but 'spin down' at $m_{1}^{\prime}$, which we encode by assuming that $U p$ is settled true at $m_{1}$, but settled false at $m_{1}^{\prime}$. We can envision three speech acts.

At $m_{0}$ (whose date time is $t_{0}$ ), Sarah, with the date time, $t_{1}$, in mind, predicts spin-up by uttering Will:Up. Bill, at $m_{2}$, thinking of $m_{0}$, utters Was: Sett:Will: $U p$ as a way, perhaps, of trying to trivialize Sarah's speech act by claiming that what Sarah said was settled true when she said it, at $m_{0}$. Bill speaks falsely: Since $U p$ is (timelessly) settled false at $m_{1}^{\prime}$, it follows that Will: $U p$ was not settled true at $m_{0}$, thus making Bill's utterance settled false at the moment, $m_{2}$, of his utterance. Jack, however, also speaking at $m_{2}$, utters Sett:Was:Will:Up as a way of positively assessing the prediction Sarah made: It is now, at $m_{2}$, settled true that what Sarah said was indeed (plain) true when she said it, at $m_{0}$. Jack speaks the settled truth: Since $U p$ is (timelessly) settled true at $m_{1}$, it follows that Will: $U p$ is true at $m_{0}$ on every history passing through (not $m_{0}$ but) $m_{2}$, even though it is false that Will: $U p$ is true at $m_{0}$ on every history passing through $m_{0}$ itself. So Was: Will: $U p$ is settled true at Jack's moment of speech, $m_{2}$, and he would be right in crediting Sarah with having spoken truly. (It turned out that she spoke the truth.)

(We refuse to take the blame for the complications revealed by disentangling this example. Blame them instead on the situation, not the reporters.)

Note, incidentally, that when 'Was:' in the above passages is evaluated at $m_{2}$, it takes us back via '<' to some moment in the causal past of $m_{2}$ (either $m_{1}$ or $m_{0}$ ), not just to some moment such as $m_{1}^{\prime}$ whose date time is in the past of the date time of $m_{2}$ ('the temporal past', as we might say).

We can also use $A t_{t}$ : to speak of contemporaneous 'might-have-beens', for example $A t_{t_{i}}: U p$, but also Was:Poss: $A t_{t_{1}}: \neg U p$. Or even, using date times exclusively, with $m_{2}$ as moment of context (and moment of evaluation), $A t_{t_{i}}: U p$, but also $A t_{t_{0}}:$ Poss: $\left._{A} t_{t_{1}}:\right\urcorner U p$.

The often misunderstood point is that one cannot escape dealing with indeterminism by substituting pure date time language for tenses.

\section{Identifying the Saunders-Wallace Proposed Semantics}

Unfolding all of the above may be belaboring the obvious, but it seems necessary as a background for becoming clear on the semantics that Saunders and Wallace say they propose since their presentation is extremely condensed.

Here are a number of short passages that hint at something like semantic rules. 
If persons are continuants [which Saunders and Wallace endorse], we do better to attribute thoughts and utterances at $t$ to continuants $C$ at $t$. That is, thoughts or utterances are attributed to ordered pairs $\langle C, t\rangle$ or slices of persons $\langle C, S\rangle, S \in C$, not to temporal parts $S$. (p. 295)

Here one might make a case for a variety of different semantic rules, but the one we are interested in is this: the word 'I' refers to the speaker of any sentence in which it occurs. (pp. 295f.)

[The authors understand this in such a way that] the referent of 'I myself', thought or uttered at time $t$ (at temporal part $S$ ) is the continuant who thinks or utters the phrase [in question]. (p. 296)

To conclude: if as Lewis proposes [and Saunders and Wallace seem to endorse] in cases of personal branching we say there are two persons present even before the branch, it is at least somewhat natural to attribute two sets of thoughts to those persons; in the case of worlds branching, it becomes entirely natural. (p. 303)

If this is depersonalized, or if branching-time theory is personalized, this sounds rather like our understanding of branching time. In particular, the attribution of utterances to continuant slice pairs $\langle C, S\rangle$ seems just like our attribution of truth to moment history pairs $\langle m, h\rangle$ as the index of evaluation. ${ }^{25}$ In further analogy to (or endorsement of) the Saunders and Wallace scheme, we allow that even before a branch point $m$, the various histories $h$ containing $m$ must be kept distinct.

When one is semantically precise, however, it becomes obvious that in contrast to Saunders and Wallace, one must distinguish (plain) truth at a moment history pair $\langle m, h\rangle$ from settled truth at a moment (the histories having been canceled by universal quantification). The next step is one of application (or metaphysics?): We submit that an utterance is, when properly idealized, a momentary event, so that

\section{Abigail uttered 'I will survive'}

is either settled true at a moment, $m$, or settled false. Its truth value (unlike the truth value of her future-tensed utterance, 'I will survive') does not depend on what happens in the future! Furthermore, although 'thoughts' (contents of thinkings) may have exotic relations to the world of events, the same is not

25 This understanding of the passage presupposes suppressing the context moment. On a different reading of the quoted passage, they might be taken to say that the context of an utterance, rather than the index of evaluation, is given via a pair $\langle C, t\rangle$. This would amount to taking the context to specify a 'history of the context', i.e., naive Occamism with a 'thin red line'. This position is completely alien to EQM which stresses the inherent symmetry of the branches and explicitly excludes taking one of them to be 'more real' than the others (Greaves and Myrvold [2008]). Thus, the more likely reading of the quoted passage is the one in terms of an index of evaluation. 
true of thinkings. Like an utterance, a thinking is properly idealized as a momentary event whose occurrence does not depend on future happenings. Yes, a thinking may be properly attributed to a continuant moment pair, but it is a settled feature of the continuant at that moment, not dependent on following the continuant for years and years through a particular possible future life history. A corollary is that a thinking, though having a fixed past, may have multiple individually possible but pairwise incompatible events in its 'future of possibilities' just like any other event.

\section{Conclusion}

So far, we have reviewed 'branching time with date times' semantics, especially in application to double time references (BTDT DTR), and critiqued the branching semantics hinted at by Saunders and Wallace. We have found points of positive contact, but have also complained about its lack of rigor and about points at which we think it takes a wrong turn. The question of giving an EQM-friendly semantics based on branching still seems to be open, however, even though some boundary conditions have been marked. How could one proceed to resolve the issue? With regard to the alternatives laid out in Section 5, FF argues in detail against all the alternatives except (2b2), the BTDT DTR semantics. An argument in favor of BTDT DTR semantics can be found in DTR. While this argument is mostly based on considerations of actual language use, it does not consider quantum physics explicitly, and some parts of it might be considered 'metaphysical'. Saunders and Wallace explicitly denounce any metaphysical motivation on the grounds of their methodological choice of a 'naturalized metaphysics'. ${ }^{26}$ We think that considerations of physics alone still point to a BTDT DTR semantics, which deviates least from the authors' choice of a representation of individuals as history-thin worms, and settles the important issue, left open in their paper, of which role a history parameter is assumed to play in their framework. Indeed, BTDT DTR semantics is the only one on which the uncertainty about the future that lies at the basis of the 'incoherence problem' is adequately represented and resolved: On this semantics, an utterance of, say, 'the outcome of the experiment will be spin up' can be assigned a settled truth-value after the experiment (it will be literally true, on the proposed semantics, to say that it is settled that it was true), but as of before the experiment, there is no settled fact of the matter as to which way it will turn out. We may conclude, following Prior ([1968], Chapter 4), that it is a simple corollary of indeterministic

26 Just a short comment on such methodology: We believe that taking that project seriously would mean to back it via empirical data about physicists' actual language use and about their actual stance toward the uncertainty faced in quantum-mechanical experiments. Anecdotal evidence is not enough to settle the issue. 
branching that it is impossible to know, at $m$, some proposition that is not settled true at $m$ (FF, Section 2B.10). Thus, we can make rigorous sense of the troubling fact that triggered the incoherence problem of EQM: We may be in a state such that all that is to be known is known, and we may still be in a state of objective uncertainty. Such is the nature of our indeterministic world.

\section{Acknowledgements}

The authors acknowledge support by the Deutsche Forschungsgemeinschaft (German National Science Foundation), MU 1816/4-1.

Nuel Belnap Department of Philosophy, University of Pittsburgh 15232 Pittsburgh, PA, USA belnap@pitt.edu

Thomas Müller Department of Philosophy, University of Utrecht 3584 CS Utrecht, The Netherlands Thomas.Mueller@phil.uu.nl

\section{References}

Belnap, N. [1992]: 'Branching Space Time', Synthese, 92, pp. 385434.

Belnap, N. [2002]: 'Double Time References: Speech Act Reports as Modalities in an Indeterminist Setting', in F. Wolter, H. Wansing, M. de Rijke and M. Zakhar yaschev (eds), Advances in Modal Logic, Volume 3, Singapore: World Scientific, pp. 3758 .

Belnap, N. [2006]: 'Bressan's Type Theoretical Combination of Quantification and Modality', in H. Lagerlund, S. Lindström and R. Sliwinski (eds), Modality Matters: Twenty Five Essays in Honour of Krister Segerberg, Volume 53 of Uppsala Philo sophical Studies, Uppsala: Department of Philosophy, Uppsala University, pp. 3154.

Belnap, N. [2007]: 'An Indeterminist View of the Parameters of Truth', in T. Müller (ed.), Philosophie der Zeit. Neue analytische Ansätze, Frankfurt a.M: Klostermann, pp. 87113.

Belnap, N., Perloff, M. and Xu, M. [2001]: Facing the Future: Agents and Choices in Our Indeterminist World, Oxford: Oxford University Press.

Bressan, A. [1972]: A General Interpreted Modal Calculus, New Haven: Yale University Press.

Garson, J. W. [2006]: Modal Logic for Philosophers, Cambridge: Cambridge University Press.

Greaves, H. [2004]: 'Understanding Deutsch's Probability in a Deterministic Multi verse', Studies in History and Philosophy of Modern Physics, 35, pp. 42356. 
Greaves, H. and Myrvold, W. [2008]: 'Everett and Evidence', in S. Saunders, J. Barrett, A. Kent and D. Wallace (eds), Everett at the Crossroads: The Many Worlds Inter pretation of Quantum Mechanics, Oxford: Oxford University Press.

Gupta, A. [1980]: The Logic of Common Nouns: An Investigation in Quantified Modal Logic, New Haven: Yale University Press.

Kaplan, D. [1989]: 'Demonstratives: An Essay on the Semantics, Logic, Metaphysics, and Epistemology of Demonstratives and other Indexicals; with Afterthoughts', in J. Almog, J. Perry and H. Wettstein (eds), Themes from Kaplan, Oxford: Oxford Uni versity Press, pp. 481614.

Lewis, D. [1986]: On the Plurality of Worlds, Oxford: Blackwell.

Lewis, D. [1976]: 'Survival and Identity', in A. Rorty (ed.), The Identity of Persons, Berkeley: University of California Press, pp. 17 40. Reprinted, with a new post script, in D. Lewis, Philosophical papers, Vol. 1, 1983, Oxford: Oxford University Press, pp. 5577.

MacFarlane, J. [2003]: 'Future Contingents and Relative Truth', The Philosophical Quarterly, 53(212), pp. 32136.

MacFarlane, J. [2005]: 'Making Sense of Relative Truth', Proceedings of the Aristote lian Society, 105, pp. 32139

Müller, T. [2005]: 'Probability Theory and Causation: A Branching Space Times Analysis', British Journal for the Philosophy of Science, 56, pp. 487520.

Parfit, D. [1976]: 'Lewis, Perry and What Matters', in A. Rorty (ed.), The Identity of Persons, Berkeley: University of California Press, pp. 91108.

Prior, A. N. [1967]: Past, Present and Future, Oxford: Oxford University Press.

Prior, A. N. [1968]: Papers on Time and Tense, Oxford: Oxford University Press.

Saunders, S. and Wallace, D. [2008]: 'Branching and Uncertainty', British Journal for the Philosophy of Science, 59, pp. 293305.

Thomason, R. H. [1970]: 'Indeterminist Time and Truth Value Gaps', Theoria, 36, pp. 26481.

Thomson, J. [1977]: Acts and Other Events, Ithaca: Cornell University Press.

Venema, Y. [2001]: 'Temporal Logic', in L. Goble (ed.), The Blackwell Guide to Philosophical Logic, Oxford: Blackwell, pp. 203223.

Wallace, D. [2008]: 'Philosophy of Quantum Mechanics', in D. Rickles (ed.), The Ash gate Companion to Contemporary Philosophy of Physics, Aldershot: Ashgate, pp. 1698.

Weiner, M. and Belnap, N. [2006]: 'How Causal Probabilities Might Fit into Our Objectively Indeterministic World', Synthese, 149, pp. 136. 